\title{
Modelling the effect of temperature and water activity on the growth rate of Aspergillus flavus and aflatoxin production in peanut meal extract agar
}

\begin{abstract}
Aspergillus flavus is the predominant species that produce aflatoxins in stored peanuts under favourable conditions. This study aimed to describe the growth and aflatoxin production by two A. flavus strains isolated from imported raw peanuts and to model the effects of temperature and aw on their colony growth rate as a function of temperature and aw in Peanut Meal Extract Agar (PMEA). A full factorial design with seven aw levels (0.85-0.98 aw) and five temperature levels $\left(20-40{ }^{\circ} \mathrm{C}\right)$ was used to investigate the growth and aflatoxin production. Colony diameter was measured daily for 28 days while AFB1 and total aflatoxin were determined on day 3, 7, 14, and 21 . The maximum colony growth rate, $\mu \max (\mathrm{mm} /$ day) was estimated by using the primary model of Baranyi, and the $\mu \max$ was then fitted to the secondary model; second-order polynomial and linear Arrhenius-Davey to describe the colony growth rate as a function of temperature and aw. The results indicated that both strains failed to grow at temperature of $20{ }^{\circ} \mathrm{C}$ with aw $<0.94$ and aw of 0.85 for all temperatures except $30{ }^{\circ} \mathrm{C}$. The highest growth rate was observed at $30{ }^{\circ} \mathrm{C}$, with 0.98 aw for both strains. The analysis of variance showed a significant effect of strain, temperature, and aw on the fungal growth and aflatoxin production $(\mathrm{p}<0.05)$. Furthermore, both secondary models were in good agreement with the observed $\mu$ max. However, the polynomial model was found to be a better predictor of the experimental data. A similar pattern was observed in aflatoxin production but in a narrower range of temperature $\left(25-35^{\circ} \mathrm{C}\right)$ and aw $(0.92-0.98 \mathrm{aw})$. The highest production of aflatoxins was observed on day 21 at $30{ }^{\circ} \mathrm{C}$ with the aw level of 0.98 for both strains. Overall, the current findings may help in improving the mycotoxin management and intervention strategies in peanuts, especially during storage.
\end{abstract}

Keyword: Peanuts; Aspergillus flavus; Aflatoxins 